\title{
infancias: voces desde la diversidad
}

maría teresa suárez ${ }^{1}$ universidad pedagógica y tecnológica de colombia - colombia josé alfredo malagón lópez ${ }^{2}$ universidad pedagógica y tecnológica de colombia - colombia

\section{resumen}

El artículo corresponde a los avances, reflexiones y posturas teórico-metodológicas del Proyecto "Voces y pensamientos: comunicación desde la diversidad" (SGI 2195, UPTC, Tunja- Boyacá - Colombia). Presenta inicialmente una introducción que corresponde al contexto colombiano contemporáneo, especialmente ubicado en el séptimo proceso de paz que se vive en Colombia, señala cómo la escuela taller surge como un tiempo y espacio de esperanza pues hace parte del programa Nacional "Escuelas Taller de Colombia Herramientas de Paz". Los siguientes apartados, manifiestan posturas que son descentramientos construidos después de una semana de encuentro con los chicos con capacidades diversas del aula Koika, es decir, un tiempo de contextualización, que se constituye en aprendizajes vitales para las y los investigadores. Primero, con relación a las dificultades comunicativas, pues se considera, que estos jóvenes no tienen capacidades comunicativas. A pesar de eso, desde el primer encuentro se comprende que la incapacidad no es de ellos sino nuestra y del mundo para comprender su voz. Aquí se contempla la construcción de relaciones intersubjetivas con ellos, con nosotros, entre ellos y nosotros, desde la diferencia como potencia y la mirada de extranjeridad, que se debate entre su libertad y el deseo de integración. El segundo descentramiento, tiene que ver con la intención formadora para "construir el futuro" de estos jóvenes. Ante esto, la investigación, asume una postura de renuncia, es decir, no se plantea objetivos relacionados con promover su desarrollo, ni estimular habilidades, pues no se pretende cumplir con una función educadora. Sólo se quiere vivir experiencias dialógicas que impacten en su presente. El tercer descentramiento, es comprender el encuentro de diversidades, sin señalamientos externos que aseguran diferencias cognitivas, las cuales ubican en lugares de desventaja o de imposibilidad. Los investigadores evitamos conocer los diagnósticos psicológicos del grupo participante, pues no se considera vital para desarrollar el proyecto. La postura, no hay puntos de normalidad natural, pues el valor y la potencia para conversar es justamente la singularidad humana.

palabras clave: inclusión; comunicación; filosofía; infancia.

\section{infância: vozes a partir da diversidade}

resumo

O artigo trata dos avanços; reflexões e posturas do Projeto "Vozes e pensamentos: a produção da comunicação a partir da diversidade" (SGI 2195, UPTC, Tunja, Boyacá Colombia). Apresenta, inicialmente, uma introdução que trata do contexto colombiano contemporâneo contextualizado no sétimo processo de paz vivido na Colômbia. Ressalta como o trabalho com oficinas de comunicação, na escola, como parte do Programa Nacional "Escuelas Taller de Colombia Herramientas de Paz" surgem como um tempo e espaço de esperança. Em seguida, são trazidas algumas posturas e descentramentos feitos

\footnotetext{
${ }^{1}$ E-mail: mariate234@gmail.com

2 E-mail: alfredomalagonlopez@gmail.com
} 
após uma semana de trabalho com os meninos, um tempo de contextualização que se constitui de aprendizagens vitais para investigadores e investigadoras, primeiramente no que se refere às dificuldades comunicativas, pois era considerado que esses jovens não tinham capacidades comunicativas. Porém, desde o primeiro encontro, compreende-se que a incapacidade não é deles, mas é nossa, é do mundo, para escutar sua voz. Aqui se contempla a construção de relações intersubjetivas entre eles, entre nós, entre eles e nós, tendo a diferença como potência e a visão de estrangeiridade, que se divide entre a liberdade e o desejo de integração. O segundo descentramento refere-se á intenção formadora para "construir seu futuro". Frente a isso, a pesquisa assume uma postura de renúncia, isto é, não se propõe objetivos relacionados ao seu desenvolvimento ou estimular habilidades, pois não se pretende cumprir uma função educadora. Apenas se busca viver experiências dialógicas que impactem seu presente. $O$ terceiro descentramento está em compreender o encontro das diversidades para além das implicações externas que focam diferenças cognitivas e, com isso, os colocam em lugares de desvantagem e de impossibilidades. Para isso, os pesquisadores evitam conhecer os diagnósticos psicológicos do grupo de participantes, não considerado vital para o desenvolvimento do projeto. No avesso de uma "naturalização da normalidade", foca-se no valor e no poder do conversar como expressão da singularidade humana.

palavras-chave: inclusão; comunicação; filosofia; infância.

\section{childhoods: voices from diversity}

summary

The article presents the advances, reflections and theoretical-methodological positions of the Project "Voices and Thoughts: communication from diversity" (SGI 2195, UPTC, Tunja, Boyacá - Colombia). It contains an introduction that corresponds to the reflection on the seventh-peace process that was experienced in the Colombian context. It displays the historical moment of the emergence of the communication wokshops in schools as a period and space of hope, as part of the National Program "Colombia Workshop Schools Tools of Peace". The following sections manifest positions of decentralization built after a week of meeting with young people with diverse abilities at the Koika classroom, representing a time of contextualization which was vital for the learning of the researchers. First, in relation to communication difficulties, as it was considered that these young people did not have communication skills. However, from the first encounter it was clear that the disability was not theirs, but ours and the world's to understand different communication possibilities. Here the construction of intersubjective relations is contemplated, regarding the difference as a potential and the look of strangeness, which is torn between its freedom and the desire for integration. The second decentering has to do with the formative intention to "build their future". In front of this, the research assumes a position of resignation, that is to say, there are no objectives related to promoting its development, nor stimulating skills, because it is not intended to fulfill an educational function. The only intention is to live dialogical experiences that impact on the present. The third decentering is to understand the meeting of diversities without external signs that ensure cognitive differences as a disadvantage or impossibility. The researchers avoid knowing the psychological diagnoses of the participating group, since it is not considered vital to the development of the project. By avoiding a perspective of "naturalization of the normality", the focus shifts to the valuing and the power of conversation as the main expression of human singularity.

keywords: inclusion; communication; philosophy; childhood. 
infancias: voces desde la diversidad

\section{en tiempos de esperanza: a modo de introducción.}

Colombia transita por momentos que hacen historia. El país camina con expectativas, temores y esperanzas por un séptimo proceso de paz, después de vivir una historia de conflicto armado que, por casi medio siglo, ha traído destrucción, muerte, pobreza, y muchos otros efectos propios de un estado de guerra. "Uno de los conflictos armados de más larga data en el mundo". Como lo manifestó el secretario de la ONU Ban Ki-Moon, antes de asistir a la firma del acuerdo de Paz. ${ }^{3}$ Ante esta situación, surgen diferentes posturas, percepciones y acciones, incertidumbres que agobian a todo un país. Algunas en contra, como se evidencia el 2 de octubre de 2016 cuando se lleva a cabo un referéndum para que la ciudadanía expresara su aprobación o rechazo a los acuerdos que se firmaron entre el gobierno y las FARC 4 . En este plebiscito triunfa el NO, lo cual refleja los temores y la contravía de algunos pensamientos frente a un proceso de paz. Otros, por el contrario, consideramos que este momento es muy importante para el país y que lo que nos sucede es más que un negociación entre guerrilla y gobierno, es un momento para pensar en reducir desigualdades y para mejorar las condiciones de vida de los colombianos.

En esta coyuntura, se consolidan proyectos que le apuestan a nuevas posibilidades. Es el caso de las "Escuelas Taller de Colombia - Herramientas para la paz" con el apoyo del Ministerio de Cultura de Colombia, un programa introducido al país por la Agencia Española de Cooperación Internacional para el Desarrollo (AECID). En la ciudad de Tunja del departamento de Boyacá, se fortalece La Fundación Escuela Taller de Boyacá (FETB) considerada como un agente para enfrentar temas de reconciliación y de creación de nuevas

\footnotetext{
${ }^{3}$ Noticia en desarrollo presentada por los noticieros del país, donde se afirma que la Asamblea General de la ONU sesionó el martes 20 de Junio de 2017 en Nueva York con felicitaciones a Colombia por poner fin a la guerra más larga del continente americano. https:// noticias.caracoltv.com/acuerdo-final/ayudamos-colombia-poner-fin-la-guerra-mas-largadel-hemisferio-obama ${ }^{4}$ Fuerzas Armadas revolucionarias de Colombia, Grupo guerrillero de izquierda instituido en el año 1964 desmovilizado.
} 
oportunidades, una escuela dirigida a personas en condición de vulnerabilidad que se pueden formar en oficios tradicionales. En palabras de Nancy Camacho directora de la escuela taller

[...] aprender oficios implica aprendizajes que trascienden... A la Escuela Taller no le interesa informar cifras de estudiantes, sino cuántas vidas se transformaron, cuántas empresas surgieron, o sea, cuántas personas se empoderaron en su oficio y ahí se mide el éxito, nosotros lo vemos como oportunidades para vivir, para salir de la violencia y contribuir con esa paz tan anhelada por el país. (Información Verbal en Entrevista I.[4 de mayo de 2017] Entrevistadora: María Teresa Suárez Vaca. Tunja. )

El Proyecto Educativo de la Escuela plantea en su visión la expectativa de contribuir en las transformaciones sociales del entorno, promoviendo procesos para la creación de sentidos de vida desde la cultura, el arte y el patrimonio, recuperando la memoria de los saberes tradicionales, para la preservación del patrimonio tangible e intangible de la región. Una condición para ingresar a estudiar en la escuela es estar en condiciones de vulnerabilidad. Por lo tanto, su población se podría considerar en cierto estado de indefensión, a nivel económico, social o político, como se refleja en las anotaciones del cuaderno de campo de esta investigación:

algunos de ellos vienen desplazados de otros lugares del país, [...] desmovilizados de grupos, [...], niños abandonados que viven en albergues, o niñas adolescentes embarazadas, [...] en situación de pobreza $[\ldots]$, que necesitan ser incluidos [...]. Son situaciones borde, situaciones límite, unas condiciones como si les arrinconaran o les empujaran hacia un abismo, eso se percibe [...] En medio de su dureza están en una condición fragilidad, es fácil, caer en las drogas, en situaciones de explotación (SUÁREZ, M. Comunicación personal, diario de campo N 2-7 mayo de 2017).

La Escuela abre sus puertas y efectivamente la confluencia es muy diversa. Algunas personas no quieren, ni reconocen límites, porque confunden los límites con el maltrato, persecución o exclusión. Entonces, el reto, además de aprender un oficio, es el de creer en sí mismo, de exigirse al máximo para sí y no por los demás o para cumplir al otro. Se evidencian situaciones como la adicción a sustancias psicoactivas, consumo de alcohol, participación en redes del micro tráfico, recurrencia a medios de subsistencia oscuros derivados del narcotráfico y del tráfico de armas, algunos de los males comunes que aquejan el país. Pero estas 
situaciones en la Escuela Taller se comprenden como actitudes efecto de las difíciles condiciones emocionales, sociales y económicas que padecen.

\begin{abstract}
Al hablar de educación en la diversidad, se está haciendo referencia a la discusión sobre cómo educar en la heterogeneidad, pues es evidente que siempre se ha educado a sujetos diversos, pero con un propósito de homogeneizar creencias y saberes, entonces lo que ha hecho diversa la educación no es sólo el hecho que las sociedades se hayan hecho diversas, de hecho, siempre lo fueron, lo que la lleva a ese punto es la decisión de las mismas sociedades y sus proyectos de constitución social. (BAQUERO, 2017, p.5).
\end{abstract}

Dentro de esta población tan particular (grupo de personas consideradas en estado de vulnerabilidad ), a la Escuela llegan también jóvenes con capacidades diversas, la gran mayoría consideradas en estado de vulnerabilidad, por sus condiciones sociales y económicas. Allí asumen el reto de trabajo y su vinculación a participar en la formación en oficios lo cual es una dinámica muy novedosa. Las personas en condición de discapacidad intelectual generalmente se ven afectadas por diversos factores, como: sociales, de rechazo y señalamiento, jurídicos, al considerarlos como trastornados mentales o inmaduros psicológicos, educativos, como pretender su homogenización. Por lo que generalmente, se les sitúa en el lugar del cuidado y la protección.

La vulnerabilidad es una condición constitutiva de los seres humanos relacionada con la posibilidad de ser lesionado. Biológicamente somos seres neoténicos, es decir, incapaces de sobrevivir por sí mismos al momento del nacimiento. [...] A su vez esta condición de vulnerabilidad, de dependencia, es esencial para la construcción de la subjetividad, la identidad y los vínculos afectivos. Así la vulnerabilidad, la interdependencia son elementos constitutivos de lo humano y debería tener una valoración positiva dentro de la sociedad. (BARRIOS, 2013, p.8).

De tal forma, es de humanos, ser o sentirse en condiciones de vulnerabilidad, es decir, no es una situación sólo de las personas con discapacidad sino es un estado posible de la humanidad. Sin embargo, estas condiciones diversas requieren una mirada especial desde la perspectiva de vida, entendiendo que han sido vulnerados y usurpados en sus derechos y por eso les deben ser restituidos, entre ellos el derecho a la educación, a la recreación, a la participación en la vida cultural y las artes, especialmente cuando son menores de 18 años o cuando los códigos los consideran -desde la vulnerabilidad- "diagnosticados" como inmaduros psicológicos o con trastorno mental. 
La Escuela Taller asume y comprende esta situación especial y acoge a estos jóvenes, la gran dificultad manifestada es "la comunicación" y la falta de recursos. Ante esta situación, la Escuela participa en una convocatoria del gobierno de Corea, solicitando apoyo especializado, y efectivamente, la Agencia Internacional de Cooperación de Corea (KOICA) se muestra interesada en apoyar el proyecto y ofrece un apoyo económico y un profesional de educación especial. Después de cumplir muchos protocolos técnicos, y de seguridad, llega Ana ${ }^{5}$, directamente de Corea, quien con la directora de la Escuela y el coordinador pedagógico elaboran el proyecto de construcción de un aula-taller para educación especial, denominada "sala Koica", la cual se adecuó con implementos y recursos especializados para trabajar con esta población.

Con Ana, a pesar de no entendernos por el idioma, (pues fue necesario conseguir traductores en la universidad), se organizó un proyecto orientado a la inclusión económica, es decir, Koica no es una guardería de niños diferentes, es un proyecto para que ellos desarrollen habilidades motrices, manuales, intelectuales, que les permitan manipular, pensar con los dedos o como queramos decirlo y ojalá generarse un sustento a través de cosas que ellos puedan transformar (Información verbal brindada por Nancy Camacho directora de la Escuela Taller de Boyacá, Entrevista I.[mayo 4 de 2017] ).

El proyecto es una propuesta de inclusión para el trabajo y el desarrollo humano, una experiencia que busca autonomía y pretende la inserción laboral y social de estos jóvenes considerados con capacidades diversas. El gran problema "la comunicación de ellos obviamente con su nueva profesora coreana". Por su parte, Ana tuvo una enorme dificultad para aprender español, y prácticamente no lo aprendió. Ella se comunicaba de otras maneras con los jóvenes, pues tenía experiencia en el trabajo con niños sordos y eso significó construir otros lenguajes para dialogar con niños autistas o con los jóvenes con discapacidad cognitiva. De alguna manera, ella se incluía como una persona más en estado de vulnerabilidad que se unía a un grupo con problemas en su comunicación.

\footnotetext{
${ }^{5}$ ANA, fue el nombre elegido por los compañeros para establecer comunicación, pero su nombre verdadero es Wookyo Joun
} 


\section{comunicación una experiencia de relación intersubjetiva}

El proyecto "Voces y pensamientos: comunicación desde la diversidad" se vincula a la Escuela Taller y pretende trabajar sobre los procesos de comunicación en la sala Koica desde la perspectiva que vincula la filosofía y la infancia.

La primera fase del proyecto, que permitió hacer una contextualización, descentra primero la inconformidad comunicativa. El primer hallazgo y aprendizaje es, que estos jóvenes no tienen incapacidades comunicativas, la incapacidad es nuestra, de los profesores y del mundo, para comprender su voz, sus formas comunicativas. Al igual que Ana, la incomprensión del idioma coreano dificultó su relación con los compañeros, pero no con los jóvenes de la sala Koica. Ella demostró que su condición de extranjera es una potencia y no una debilidad, pues construyó lenguajes que le permitieron establecer relaciones especiales con sus estudiantes. Por lo tanto, Ana y los jóvenes del aula Koica son extranjeros en un mundo que no les comprende.

Walter Kohan (2011) manifiesta que existe variedad de conceptos y sentidos sociales construidos frente a un extranjero: los turistas, inmigrantes, huéspedes, los que luchan causas ajenas fuera de su país de origen, los exiliados, peregrinos etc, pero un concepto dominante de extranjero, lo relaciona con otra nacionalidad, una lengua, una tierra y algunos otros sentidos,

Extranjera puede ser una figura que no viste nuestra ropa, que no piensa nuestro pensamiento, o, de manera menos estricta que vive otra vida. Así, el extranjero, de manera general, es alguien que está instalado fuera de "nuestro" universo de la normalidad (KOHAN, 2011, p.15).

Bajo esta mirada, Ana y el grupo de jóvenes, se constituyen en extranjeros que no encajan en la normalidad de nuestro universo tunjano ${ }^{6}$, pues no hablan nuestra lengua, se diría que son incapaces de comunicarse, de entendernos a los "normales". Estas percepciones, transgreden no sólo las fronteras comunicativas, sino también la concepción como sujetos diversos. Se dice con naturalidad que son niños diferentes, especiales, que no pueden pensar, saber y vivir como adultos, todo, porque su lenguaje es diferente.

\footnotetext{
${ }^{6}$ El universo Tunjano hace referencia al grupo social y cultural, que se moviliza cotidianamente en la ciudad. Generalmente, se piensa de forma homogénea, lo que invisibiliza la diversidad.
} 
Los imaginarios sociales y culturales subrayan dificultades comunicativas en las personas con discapacidad, como un problema de un colectivo minoritario, pero la pregunta por el cómo la sociedad ha intentado entender los lenguajes particulares de esta población, es un interrogante de pocas respuestas, pues estos lenguajes han sido desconocidos y subvalorados. Para Skliar y Tellez (2008, p. 116), se ha privilegiado "mucho más el ser capaces de hablar «sobre la diversidad», «sobre el otro», «acerca del otro» que poder «conversar con el otro»y, aún mucho menos, la de dejar que «los otros conversen entre ellos mismos»".

Limitados son los espacios para el diálogo con y para las personas en condiciones diversas, se desconocen sus pensamientos, su comprensión del mundo, se desconoce su voz. Por lo tanto, sin espacios para escuchar y dialogar, la inclusión social se hace improbable y los discursos sobre la garantía de sus derechos, se tornan vacíos.

El proyecto voces desde la diversidad es una propuesta en perspectiva de filosofía e infancia (SUÁREZ; GONZÁLEZ, p. 2017) que crea espacios de diálogo y de escucha, conversaciones que requieren interacción, tiempos de intersubjetividad que permiten conocer y hacer visible el pensamiento de las personas que se encuentran a través de diferentes lenguajes y del reconocimiento de su singularidad, con el ánimo de contribuir a que su participación no se restrinja a la formación de mano de obra o al aprendizaje de un oficio, sino también considere la toma de decisiones dentro de la comunidad.

El estado de extranjeridad no necesariamente es un estado de indefensión o de carencias. Tiene potencias infinitas en un proyecto que vincula la filosofía y la infancia y que invita a percibir todo como novedoso, a preguntarse por lo desconocido, por la vida misma. La filosofía implica una capacidad de asombro y duda permanente. La extranjeridad y la infancia contemplan la novedad y lo desconocido como potencia. Para los "normales" y para los "residentes" el trabajo es sobre la estabilidad y lo cotidiano, que hacen ver que todo está en orden, sin novedad. Aquí, la mirada y el vivir se vuelven rutina. El estado de extranjeridad se identifica con una concepción de infancia, que no la concibe atrapada en unos 
límites de edad, sino que la considera una condición especial de la experiencia humana y una forma diferente de habitar en el mundo, una disposición que transita de forma heterogénea, desacompasada, variable, mutable, en mucha ocasiones sin lógica. La infancia se aproxima a la condición de extranjeridad al ser huidiza, inaprensible, disconforme, diversa, difícil de encasillar (CELY; ESPINOSA; SUÁREZ, 2016).

El proyecto considera que es tiempo de escuchar la voz de las infancias que se materializa en los jóvenes que llegan a la Escuela Taller de Boyacá, de sacar a la luz sus pensamientos, de que el mundo ponga de su parte para comprenderles, pues hay desconocimiento, sobre su percepción de la vida y sobre su subjetividad, es momento para dialogar, preguntar, escuchar y comprenderles, no con la pretensión arrogante de darles la oportunidad de expresar sentimientos, sino con la disposición de recibir sus verdades y de reconocer su existencia en nuestras vidas.

Larrosa (2008, p. 1) señala "el aula como lugar de la voz. Y la voz, [...] como la marca de la subjetividad en el lenguaje". La voz no es sólo el sonido generado por el aparato fonador humano, o la voz gramatical, como diátesis asociada con el verbo, o la palabra, en cuanto vocablo o unidad léxica preparada para el arte de confeccionar diccionarios. La voz aquí, tiene significado, es sentimiento, experiencia, vida, es una parte del mismo ser. Larrosa manifiesta que el lenguaje lleva una marca que transmite y que esta se encuentra ligada a la experiencia del que habla y a la experiencia del que escucha.

La voz es esa marca, esa experiencia, esos avatares que hacen que los que hablan y los que escuchan, los que dan y los que reciben, sean unos sujetos concretos, singulares y finitos, de carne y hueso, y no sólo máquinas comunicativas (emisores y receptores de significados) o máquinas cognitivas (codificadores y decodificadores de información). (LARROSA, 2008, p.2)

Entonces, la voz no es mecánica ni está reducida a expresiones comunes que se dicen sólo por cortesía, pero sin sentimiento. La voz es la cara sensible de la lengua que encierra algo más que significados. Pensar y reconocer la existencia de la voz, desde esta perspectiva, da un sentido especial para el proyecto que llevamos adelante, pues lo que se pretende es motivar la producción, la escucha y 
la comprensión de las voces de estas personas consideradas diversas. La voz se puede escuchar, leer, ver, sentir. Son múltiples los lenguajes que se pueden utilizar para sacar a la luz la voz del pensamiento. Ante esto, pierde importancia el sonido imperfecto generado por el órgano fonador. Un nudo en la garganta, posiblemente tartamudo, gritado, entrecortado o mal pronunciado habla de una experiencia. El valor lo tiene la relación subjetiva de quien lo expresa con lo que expresa (ira, enojo, amor, esperanza, desesperanza, etc). Es la voz de su propio ser, que comunica. En conclusión, no se mira a esta población con problemas de comunicación, la problemática se traslada y se une a la reivindicación y al reconocimiento de su voz.

\section{viviendo en el presente}

El segundo descentramiento que se vive, y que se transforma después de la contextualización, tiene que ver con la intención inicial del proyecto de investigación, un propósito formador y de estimulación en los procesos de comunicación de los jóvenes, evidentemente, se unía a las intenciones educativas para "construir su futuro". (expresión tomada del Proyecto Educativo, que evidencia algunas orientaciones construidas y estipuladas por la Agencia Internacional de Cooperación de Corea). Gran lema educativo que alude a formar, desarrollar, estimular, preparar para la vida. Cientos de buenas intenciones a largo plazo y/o de miradas que se proyectan en el orden de lo teleológico, pues a esos alumnos se les considera incapaces de construir su propia vida. Las instituciones, en general, entran en relación con ellos a partir del diseño de actividades para su formación y, en muchos casos, implementan acciones que buscan normalizarlos, sin tener en cuenta sus diversas capacidades y mucho menos sus intereses.

Estas acciones restrictivas y la necesidad imperiosa de definir su futuro se sustenta en su supuesta incapacidad para dar dirección a sus propias vidas. En las prácticas y en el lenguaje de los profesionales se evoca el asistencialismo, en las relaciones de las personas de la comunidad y en los pasillos de los edificios se cuela el aroma y el recuerdo de aquellos sanatorios de otros tiempos. (FORERO, 2015. p.21)

Esto refleja una preocupación, de alguna manera, sobreprotectora, un afán por el mañana, por su futuro y no por su presente. La obligatoriedad de asistir al 
colegio, es pensando en su propio bien. "Hasta donde pueda llegar", se dice como consuelo ante esas vidas que se perciben como deficientes. No está en juego su pensamiento y su sentir en el acto de ir a la escuela. No es casual que, al dialogar con estos jóvenes, se encuentren sentimientos frustrantes frente a la experiencia escolar, señalamientos que para ellos son molestos, incómodos. Su voz refleja una carga emocional dolorosa. Son conscientes de su condición, se han apropiado de los rótulos hechos por otros, están convencidos de su discapacidad y saben que esto es un problema para el profesor y la institución, se sienten menospreciados por los compañeros y, en algunos casos, por los mismos profesores. Comprenden claramente el rechazo y se sienten agobiados por los señalamientos. En el registro de la conversación sostenida en el segundo encuentro de la fase exploratoria, se escuchan diversas expresiones donde el grupo de jóvenes manifiesta su sentir así:

el profe dijo, este joven no avanza igual que los otros, es un niño especial,
que problema!! Mandémoslo con la psicóloga" [...] nos hacen bulling... o
sea se la montan a uno,[...] "nos dicen especiales, bobos y cuando hay
pelea un man me gritó: retrasado!!". [...] eso es matoneo, [¿qué es
matoneo?] pues que ofenden, que lo maltratan y se burlan" [...] uno tiene
que hacerse el bobo, que todo lo que digan pues... resbale!!! (SUÁREZ,
M. comunicación personal, diario de campo 3.14 mayo de 2017).

Estas voces, son gritos de inconformidad, sentimientos dolorosos por las situaciones que rodean a estos jóvenes y muestran el precio de estar inmersos en una sociedad que no comprende su diversidad. El derecho de igualdad, de estar incluido se convierte, así, en una experiencia de desigualdad, de violencia, de sometimiento, todo bajo una noble intención educativa, que muchos no comprenden, pues por pensar en su futuro, no se está pensando en su presente.

Ante esto, la experiencia investigativa asume una postura de renuncia frente a la intención formadora, es decir, re plantea los propósitos relacionados con promover el desarrollo de los jóvenes, y de estimular habilidades. Por lo tanto, no pretende cumplir con una función educadora, en el sentido de preparar para el futuro, sino que sólo se quiere vivir experiencias dialógicas que impacten en su presente. Pues, como plantea Forero (2015, p. 21), "la formación como proceso surge como proceso interior, proceso en constante desarrollo y progresión, no se plantea como un objetivo, pues es el recorrido en sí mismo el que va construyendo 
al sujeto". Este modo de caminar permite hacer construcciones propias, sin la tensión que produce esperar avances o desarrollos a cambio. Esto implica romper con los rótulos clínicos y sociales ¿Quién podría determinar una manera óptima de ser, un límite de lo que somos o un estándar del "ser" humano? Esta postura es extraña, pero liberadora, pues la preocupación finalmente es escuchar a los jóvenes, movilizar pensamientos sobre sus propios pensamientos con el fin de hacer filosofía, no como experiencia de desarrollo sino como ejercicio de vida.

\section{un escenario de diversidades}

La exigencia central de estos encuentros, es de reconocimiento, de escucha, sin juzgamiento frente a lo diferente o lo diverso visto como algo negativo o como algo en desventaja, un encontrarnos de manera intersubjetiva con proximidades vitales, de amistad, fraternidad, hospitalidad. Un encuentro entre extranjeros que se afectan y reconocen mutuamente, sin caracterizaciones o diagnósticos clínicos que señalan imposibilidades. Este, sin duda, es el tercer descentramiento, que saca de las lógicas comunes, un encuentro de diversidades, sin señalamientos externos que aseguran diferencias cognitivas, las cuales ubican en lugares de desventaja o de imposibilidad. Los investigadores evitamos conocer los diagnósticos psicológicos o médicos del grupo participante, pues no se consideran vitales para desarrollar el proyecto. Sostenemos que no hay puntos de normalidad natural, pues el valor y la potencia para conversar es justamente la singularidad humana. Una experiencia que como manifiesta Skliar (2017, p. 20)

[...] debería ser el sentido del gesto-acto del educar: recibir al otro, sin cuestiones, sin preguntas, sin sospechas, $\mathrm{y}$, sobre todo, sin juzgar, para entablar una conversación a propósito de qué haremos con el mundo y qué haremos con nuestras vidas.

En estos encuentros cotidianos, se proponen diálogos, se invita a conversar de la vida, de las propias historias que evocan sentimientos, de las múltiples verdades construidas. Es decir, se abren caminos de alteridad, donde la multiplicidad, los pliegues, los bordes, las dudas, tienen cabida, pues no hay nada definido ni negado. Entonces, todo termina siendo potencia. Por lo tanto, se abre la posibilidad para construirse o construirnos, es decir, ser a través del otro, donde 
ese otro, pese a no ser igual (y gracias a ello), motiva o acompaña la configuración de un nuevo ser en movimiento, en constante cambio. Aquí el maestro es parte de ese derrotero, pues, "la tarea del maestro sigue siendo, siempre lo ha sido, la de ser y provocar alteridad" (SKLIAR, 2017, p. 160). La pretensión es construir espacios de hospitalidad, sin agredir la extranjeridad, para que las voces sean escuchadas y los pensamientos sean reconocidos. Aparece así la oportunidad de conocer la dimensión subjetiva de estos extranjeros, de conocer y reconocer sus pensamientos, su modo de ver el mundo y su vivir, teniendo en cuenta que:

Las prácticas de los niños y jóvenes de hoy son prácticas de experiencia de orden individual y grupal, donde se reconoce y reconstruye el cuerpo y el espíritu al tiempo, donde el dualismo, otrora determinante en las maneras de ser y de actuar, se desdibuja, donde la paradoja de lo plural es una alternativa a lo individual, donde opuesto a las formas modernas de identidad, los niños y jóvenes de hoy apuestan por "identificaciones múltiples", siempre se es otro, esto 'implica acudir al juego de las máscaras, la duplicidad donde se encuentra el germen de las resistencias, de las subversiones, de aquellas que no son resistencias políticas, sino que caracterizan las prácticas juveniles' Este es precisamente el lugar para la diferencia y lo heterogéneo que permite el aprender a pensar. (PULIDO, 2004, p. 11).

En conclusión, la propuesta es caminar fuera de la rutina con los sentidos dispuestos para ver el mundo desde otras posibles y poder contemplar diversas perspectivas. Para esto, se requiere una atenta receptividad desde la escucha, que va más allá del oír, pues implica una disposición multisensorial para percibir lo que acontece, lo que se presenta. Se requiere también estar "atento" - en sus dos acepciones: de fijar la atención y de ser cortés o comedido -, es decir, estar presto al llamado del otro, a su voz, su sentir y su pensar, desde su extrañeza para hacerlo más prójimo, más cercano, más vecino, con la vulnerabilidad propia de quienes sentimos la humanidad. Por último, se requiere una disposición para conversar, en este sentido.

Creo que conversar es un gesto diferente, un encuentro sin principio ni fin que se desvía, avanza y retrocede, se pierde y quizá se reencuentra. Pienso más en las políticas de la amistad, de la fraternidad, de una conversación que no se sostenga en el "porque yo lo digo" sino en el encuentro entre dos o más fragilidades. (SKLIAR, 2017, p. 173)

Estos son los primeros caminos y conclusiones construidos, que fueron vitales para el proyecto "Voces y pensamientos: comunicación desde la diversidad" y que orientarán las perspectivas metodológicas para los futuros 
encuentros con los jóvenes de la Escuela Taller de Boyacá. Continúa, la expectativa y el desafío por la construcción de posturas que descentren las lógicas comunes. Continúa también la preocupación por construir no canales de comunicación sino de escucha a partir de la experiencia filosófica que permita conversar entre extranjeros, sin pretender que salgan de su mundo y acogerlos respetando sus y nuestras condiciones diversas, una experiencia de vida que se preocupa por el presente, por un estar bien, sin expectativas posteriores.

\section{referencias}

BAQUERO, Partrick. Educación para la Diversidad: ética del reconocimiento. Periódico Desde la U. n. 46, UPTC, mayo/2017.

BARRIOS, Miguel. Crianza en condiciones de exclusión, marginalidad y violencia social en el centro de Bogotá. 2013. Tesis Doctoral. Universidad Nacional de Colombia. Bogotá: 2013.

CELY, Adriana; ESPINOSA, Ingrid; SUÁREZ, María Teresa. Filosofía y literatura: Una experiencia de encuentro con la infancia. Quaestiones disputatae. v.9 n. 19, p. 28-43, 2016.

FORERO, María.; ORJUELA, Gloria.; PEREA, Francisco.; CRUZ, Alexandra. Modelo ecológico: Educación y Trabajo con personas con discapacidad intelectual. Bogotá: Universidad Pedagógica Nacional, 2015.

KOHAN, Walter. Filosofía y educación: la infancia y la política como pretexto. 2. ed. Caracas: Fondo editorial Fundarte, 2011.

LARROSA, Jorge. Aprender de oído -2008-disponible em:

<https:// documentop.com/aprender-de-oido_5990b3b31723ddddb6d7ac0f.html>.

Acceso: diciembre 01 de 2017.

PULIDO, Oscar. Del enseñar conceptos como aventura del pensamiento: la filosofía, la pedagogía y los niños. Cuestiones de Filosofía. n. 6, Tunja: Universidad Pedagógica y Tecnológica de Colombia, 2004, p. 31-36.

SKLIAR, Carlos; TÉLLEZ, Magaldy. Conmover la educación: Ensayos para una pedagogía de la diferencia. Buenos Aires: Noveduc, 2008.

SKLIAR, Carlos. Pedagogías de las diferencias. Buenos Aires: Noveduc, 2017.

SUAREZ, María Teresa; GONZALEZ, Bibiana; LARA, Paola. Apropiaciones y experiencias pedagógicas de filosofía e infancia en Colombia. Praxis $\mathcal{E}$ Saber. v. 8 n. 16, p. 225-246, Enero/mayo, $2017 . \quad$ Disponible en: <https:// doi.org/10.19053/22160159.v8.n16.2017.6184>.

recibido en: 28.09 .2017

aprobado en: 08.12.2017 